\title{
Migración de venezolanos a Florida Central, Estados Unidos. Aspectos relacionados con la percepción de condiciones y necesidades de salud mental en 2019*
}

\section{Immigration of Venezuelans to Central Florida, United States. Aspects of the perception of mental health conditions and needs in 2019}

\author{
Migração de venezuelanos para a Flórida Central, \\ Recibido: 09 de Diciembre de 2019. Aceptado: 27 de \\ Enero de 2020. Publicado: 1 de Octubre de 2020. \\ DOI: https://doi.org/10.11144/Javeriana.rgps19.mvfc \\ Andrés Cubillos Novella ${ }^{a}$ \\ Pontificia Universidad Javeriana, Colombia \\ ORCID: https://orcid.org/0000-0003-2943-3590 \\ Angélica María Vargas-Monroy \\ Pontificia Universidad Javeriana, Colombia \\ ORCID: https://orcid.org/0000-0002-6087-9462 \\ Tracy Wharton \\ University of Central Florida, Estados Unidos \\ ORCID: https://orcid.org/0000-0002-3734-8380
} Estados Unidos. Aspectos relacionados com a percepção de condições e necessidades de saúde mental em 2019

\begin{abstract}
Para citar este artículo Cubillos A, Vargas-Monroy AM, Wharton T. Migración de venezolanos a Florida Central, Estados Unidos. Aspectos relacionados con la percepción de condiciones y necesidades de salud mental en 2019. Rev Gerenc Polit Salud. 2020;19. https://doi.org/10.11144/Javeriana.rgps19.mvcf
\end{abstract}

\footnotetext{
${ }^{\text {a }}$ Autor de correspondencia. E-mail: cubillos.a@javeriana.edu.co
} 


\section{Resumen}

Este estudio utilizó un enfoque fenomenológico cualitativo con migrantes venezolanos ubicados en Florida Central, Estados Unidos, para explorar las percepciones relacionadas con la salud mental. Se obtuvo información sobre las expectativas y experiencias relacionadas con su viaje, las relaciones sociales y familiares, y el contexto socioeconómico en el que se perciben a sí mismos, así como información relacionada con sus reacciones emocionales, físicas, y cognitivas antes, durante, y después de migrar. La mayoría de los participantes en el estudio adujeron razones políticas o económicas para abandonar su país de origen, pero más de la mitad, señaló como un motivo importante el futuro de sus hijos. Cuatro de cada cinco personas migrantes indicaron estrés, y tres de cada cinco reportaron una tristeza o frustración significativa y manifiestan que no tienen control sobre su realidad actual, y cuatro de cada cinco participantes informaron sentimientos de aislamiento desde su llegada a Florida. El aislamiento es un predictor importante de los desafíos de salud mental, y la falta de apoyo en las redes sociales está relacionada con la resiliencia limitada.

Palabras clave: Migrante, salud mental, Venezuela, Estados Unidos.

\section{Abstract}

This study used a qualitative, phenomenological approach to engage Venezuelan immigrants to Central Florida, United States, to explore perceptions related to mental health. Information obtained focused on the expectations and experiences related to their journey, social and family relationships, and the socioeconomic context in which they currently perceive themselves, as well as information related to their emotional, physical, and cognitive reactions before, during, and after their journey. The majority of participants in this study described political or economic reasons for leaving their country of origin, and for more than half, motives related to future conditions for their children. Four out of 5 immigrants indicated stress, and 3 out of 5 reported significant sadness or frustration and feeling that they had no control over their situation. Out of 5 participants reported feelings of isolation since arriving in Florida. This is an important insight, as isolation is an important predictor of mental health challenges, and lack of social network support is related to limited resiliency.

Keywords: Migrant, mental health, Venezuela, United States.

\section{Resumo}

Este estudo utilizou uma abordagem fenomenológica qualitativa com migrantes venezuelanos localizados no centro da Flórida, Estados Unidos, para explorar percepções relacionadas à saúde mental. Foram obtidas informações sobre as expectativas e experiências relacionadas à sua viagem, relações sociais e familiares e o contexto socioeconômico no qual estão inseridos, bem como informações relacionadas às suas reações emocionais, físicas e cognitivas antes, durante e após a migração. A maioria dos participantes do estudo descreveu razões políticas ou econômicas para deixar seu país de origem, mas mais da metade indicou o futuro de seus filhos como um motivo importante para a migração. Quatro em cada cinco migrantes indicaram estresse, e três em cada cinco relataram tristeza ou frustração significativa e declararam não ter controle sobre sua realidade atual, e quatro em cada cinco participantes relataram sentimentos de isolamento desde a chegada à Flórida. O isolamento é um importante preditor de desafios à saúde mental, e a falta de apoio nas redes sociais está relacionada à resiliência limitada.

Palavras-chave: Migrante, saúde mental, Venezuela, Estados Unidos. 
Migración de venezolanos a Florida Central, Estados Unidos. Aspectos relacionados con la percepción de condiciones y necesidades de salud mental en 2019

\section{Introducción}

El actual éxodo venezolano es uno de los mayores movimientos demográficos humanos de la historia reciente. Aunque cientos de miles de ciudadanos venezolanos han huido a otros países en busca de seguridad, oportunidad, alimentación y medicamentos, poco se sabe acerca de su salud mental o factores relacionados con la resiliencia en esta población. En este sentido, el objetivo del documento es dar a conocer resultados parciales de la investigación que se viene desarrollando en torno a la percepción de salud mental de la población venezolana ubicada en Florida Central, Estados Unidos, específicamente en Orlando, Apopka y Kissimmee. Este estudio hace parte de un proyecto multicéntrico de la Universidad Central de la Florida, EE.UU, y el Instituto de Salud Pública de la Pontificia Universidad Javeriana, Colombia. El trabajo de campo comprendió aplicación de encuestas, entrevistas en profundidad y grupos focales con población venezolana mayor de edad, cuyas percepciones buscan comprender las implicaciones en la salud mental, en el contexto de una migración que ha sido considerada como forzada, dadas las implicaciones del régimen político de Venezuela y la necesidad humana de buscar mejores condiciones de vida.

Este artículo muestra el perfil sociodemográfico, las condiciones de calidad de vida de la población migrante, las condiciones de poder adquisitivo y la movilidad social descendente que se ha presentado en esta migración, desde un contexto latinoamericano hacia un contexto considerado como desarrollado (Florida, Estados Unidos). Se pretende determinar qué tanto esta movilidad ha afectado las condiciones de salud mental de las personas, en lo que tiene que ver con el impacto migratorio. Igualmente, se analizan sus percepciones en lo referente a las condiciones mentales y a las relaciones establecidas entre redes de apoyo, condiciones de vida, relaciones familiares y sociales y contextos socioeconómicos en el destino. Igualmente se establecerán esas relaciones iniciales a la luz de migración versus el transnacionalismo, entendido este último como las conexiones sociales y familiares con el contexto de origen, y qué tanto este aspecto afecta la salud mental de los migrantes.

De acuerdo con los Objetivos de Desarrollo Sostenible 2030 (ODS) aprobados por Naciones Unidas (1), no es posible alcanzar el desarrollo sostenible sin hacer una inversión prioritaria en la salud mental de las personas. El 4,4\% de la población mundial sufre de depresión, lo que se constituye en una de las mayores condiciones de incapacidad en la población global, y es la primera causa de más de 800.000 muertes relacionadas con suicidios (2). En la agenda de las Naciones Unidas para los ODS es importante establecer estrategias de intervención que garanticen el bienestar físico y mental de la población; para lo cual es necesario proveer acceso a servicios de salud universal, cumpliendo al objetivo número 3. La prevención y la atención continua en salud mental debe ser una prioridad en todos los países, incluyendo a los migrantes, para evitar peores condiciones de salud mental (2).

De acuerdo con la Organización Mundial de la Salud (OMS), la salud mental ha sido un tema ausente en muchos planes y programas dirigidos a poblaciones migrantes (3). Según la OMS la salud mental de los migrantes muchas veces está económicamente desprotegida, dejándolos 
expuestos a riesgos de abusos, violencia, explotación, discriminación, obstáculos para acceder a servicios sanitarios y sociales y falta de continuidad en la atención (3).

El reasentamiento de las poblaciones en otros contextos ha demostrado, en muchos casos, ser un riesgo para la salud mental $(4,5)$. Las relaciones entre raza, estatus socioeconómico, país de origen, etnia y género, parecen afectar las condiciones de salud mental de las personas. Algunos estudios demuestran que los factores relacionados con el contexto tales como el vecindario a donde llegan los migrantes, las conformaciones familiares, las competencias lingüísticas, la discriminación, el aislamiento social, las dificultades para emplearse y los procesos de aculturación, en muchos casos generan estrés (6) y parecen sugerir una relación directa entre migración y salud mental $(4,5,7,8)$.

A partir de la década de 1930 se pueden encontrar dos aproximaciones explicativas a la relación migración y salud mental: (i) la de causalidad, en la que se entiende la migración como un factor de riesgo de la aparición de problemas y trastornos de salud mental; y (ii) la de selección, según la que se establece que las personas con problemas de salud mental tienen más probabilidad de migrar $(9,10)$.

El modelo explicativo de la respuesta de estrés, se encuentra en la base las explicaciones en las que se entiende la migración como un factor que puede precipitar problemas de salud mental. De acuerdo con este modelo, la migración se constituye en un evento vital altamente desafiante o amenazante; convirtiéndose en una situación estresante o un estresor, que pone a prueba los recursos y la capacidad de adaptación de las personas $(5,9-11)$.

El término aculturación se refiere al proceso de adaptación de un migrante a la cultura y circunstancias de vida del país de acogida; es así como se entiende que durante este proceso se puede producir una tensión psicológica o emocional, que conlleva a una respuesta de estrés, la cual bajo ciertas características de intensidad y duración se puede asociar a ansiedad, depresión, trastornos del ánimo y dependencia de drogas o alcohol (12-16).

En un estudio cualitativo realizado con 20 migrantes en tránsito por México y con destino a Estados Unidos, se encontró que aunque los migrantes no manifestaron haber experimentado problemas de salud mental durante su proceso migratorio (antes, durante o después de la migración) concebidos como enfermedades mentales; en su discurso es posible identificar emociones, pensamientos, síntomas somáticos y cambios de comportamiento que denotan problemas de adaptación o problemas de salud mental, generalmente caracterizados por estados de ánimo temporales como angustia, miedo, desesperación, tristeza, preocupación por los familiares, impotencia, soledad, aislamiento, enojo, frustración e indecisión. Las manifestaciones somáticas expresadas más comúnmente entre los migrantes fueron pérdida de sueño, estrés, llanto, dolores de cabeza, cambios en el apetito, cansancio y fatiga (16). 
El impacto de la aculturación en los migrantes está mediado por las condiciones o características del país huésped (p. ej., similitud de la cultura de acogida con la cultura del país de origen, receptividad de la cultura huésped, diferencias de lenguaje, posibilidades socioeconómicas que ofrece el país de acogida, etc.); las condiciones de la migración (p. ej., si es forzada, por razones económicas, por ruptura de vínculos familiares y sociales en el país de origen); así como las características personales del migrante (p. ej., la edad, las características de personalidad, nivel educativo, voluntad de migrar, posición socioeconómica, conocimiento previo del idioma del país receptor) (10).

Respecto a las características del entorno de acogida, se ha identificado mayor probabilidad de presentar trastornos y problemas de salud mental entre los migrantes que perciben más brechas culturales entre su país de origen y el país huésped (14), los que perciben mayor discriminación por su estatus migrante $(9,13,14,16)$, los que encuentran mayores dificultades para encontrar trabajo $(12,14,15)$; y cuando el idioma del país de acogida es diferente al idioma de país de origen (9).

Con relación a las características personales, se ha encontrado que los niños y preadolescentes logran una mejor adaptación cultural, por lo que habitualmente el estrés aculturativo tiende a ser menor en este grupo de migrantes; lo mismo sucede con las personas que dominan el idioma del país huésped (14); por el contrario, las mujeres tienen mayor probabilidad de presentar problemas de salud mental, en comparación con los hombres (12-15); y el menor nivel educativo, se asocia a mayor prevalencia de trastornos mentales (14).

Este estudio pretende analizar las relaciones entre migración y las condiciones de salud mental de un grupo de migrantes venezolanos, buscando comprender las implicaciones que ha tenido para los venezolanos la movilidad de su país al Estado de la Florida en Estados Unidos. A pesar de la relación histórica de este país con los hispanos, parecen existir situaciones que, en el contexto de las migraciones, en lo que a salud mental refiere, las poblaciones se siguen viendo afectadas por el hecho de migrar. Para este caso particular de los venezolanos, al ser un hecho forzado, los impactos en su salud mental son un hallazgo para tener en cuenta en la atención de estas poblaciones, considerando su propio contexto, las necesidades y realidades presentes.

\section{Contexto de la migración de venezolanos al exterior}

Desde principios del siglo XXI las denominadas Misiones Bolivarianas han marcado la senda de las migraciones de venezolanos, colombianos y población desplazada que se encontraba en Venezuela a causa del conflicto armado. En el año 2000, Hugo Chávez, a través de esta estrategia buscó regularizar la estancia de muchos colombianos que se encontraban viviendo en el vecino país, con el propósito de evitar las peores condiciones de calidad de vida de estas poblaciones en el contexto venezolano. Sin embargo, esta regularización no se pudo llevar a cabo de la manera deseada, debido a la crisis económica y social que se presentó en Venezuela con la caída del precio del petróleo, que entre 2013 y 2014, junto con los cambios en las políticas migratorias implementadas por el nuevo gobierno de Maduro, generaron el inicio de una de las migraciones 
que ha impactado fuertemente el contexto latinoamericano, por la duración y el volumen de personas (17).

La baja de los precios del petróleo desde el inicio de la crisis en el año 2013, descendió un 76\%, al pasar de los 115 dólares a los 30 dólares (17). Lo anterior generó implicaciones en Venezuela y en Colombia, al tener una economía dependiente, sobre todo el punto de frontera como Norte de Santander, y a lo largo de los más de 2 mil kilómetros de frontera, cuyas poblaciones viven una interrelación económica y social desde hace muchos años.

Lo anterior generó en Venezuela una crisis económica, política y social sin precedentes que dio inicio a un proceso de movilidad internacional sin precedentes en su historia. Si bien una vez Venezuela fue considerada por muchos como el sueño latinoamericano para las poblaciones procedentes de Colombia y otros vecinos regionales, esta fue la primera vez que los venezolanos comenzaron a migrar hacia otras naciones en búsqueda de mejores oportunidades, impulsados por la creciente inestabilidad política, económica y social de su país.

De acuerdo con Migración Colombia en lo referente a la evolución de la migración venezolana al país $(18,19)$, entre 2016 a 2019 llegaron a convertirse en la migración más importante de la región, y en el contexto mundial solamente la ha superado la de los Sirios. Al mirar los datos de esta migración caracterizada principalmente por la búsqueda de asilo, podemos ver que las cifras son particularmente importantes en el caso de Colombia (figura 1).

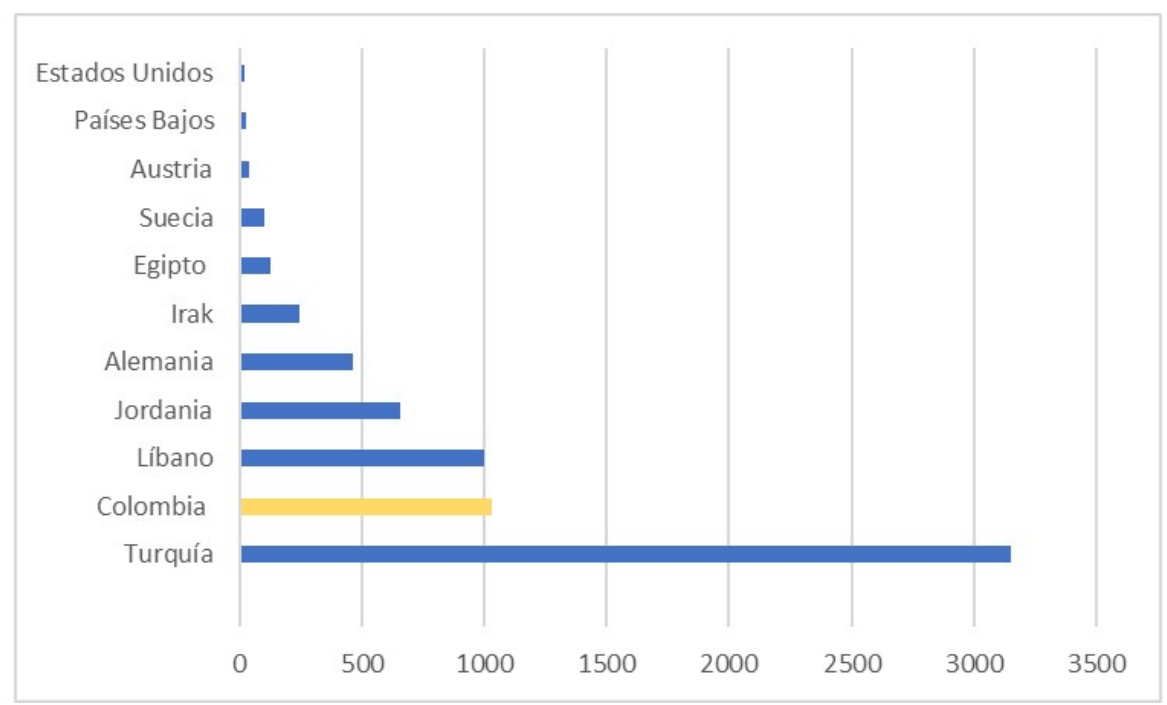

Figura 1 Comparación población migrante en Colombia con los países receptores de refugiados sirios (miles de personas)

Fuente: elaboración propia con base en información de la Estrategia para la Atención de la Migración desde Venezuela establecida por el CONPES 3950 (20) y por el Departamento de Seguridad Nacional de Estados Unidos $(21,22)$. 
Colombia ha sido el país que ha recibido más población procedente de Venezuela y la mayoría de esta población se encuentra en condición de migración o refugio; esta diferencia es muy importante, debido a que los primeros buscan mejorar sus condiciones de subsistencia, los segundos huyen del país motivados por el riesgo inminente para la vida. Esta segunda condición les otorga un estatus especial relacionado con el derecho internacional, por lo cual los gobiernos deben establecer estrategias para garantizar la seguridad de estas poblaciones.

De acuerdo con Migración Colombia y algunos de los informes que analizan esta realidad migratoria $(18,19)$, de los 2,3 millones de venezolanos que viven en el exterior 299 mil se encuentran en condiciones de asilo; el 56\% corresponde a población masculina y el $44 \%$ a población femenina. Es importante señalar que el 73,6\% se encuentra en el grupo de edad correspondiente a los 20 a 39 años (Población en Edad de Trabajar, PET) (23).

En el marco de Estados Unidos, los datos muestran que el Estado de la Florida es el que más ha recibido poblaciones provenientes de Venezuela, llegando a un total de 248 mil migrantes y, con respecto a las solicitudes de asilo, en el periodo 2014-2017 recibieron 27.579 solicitudes (22), para el año 2018 aumentaron significativamente llegando a 28.660 solicitudes (24), y para enero de 20192.064 personas habían solicitado asilo, según números oficiales de aduanas e inmigración de Estados Unidos. Aunque las cifras oficiales son difíciles de obtener, los medios de comunicación informan que sólo una fracción de estas solicitudes de asilo es aprobada, con más del 50\% fueron denegadas explícitamente (24). Estados Unidos deportó a 336 personas a Venezuela en 2018 (25). Esta población se ha ubicado principalmente en Florida Central, en lugares como Apopka, Kissimmee y Orlando, lugares que han recibido poblaciones procedentes de América Latina y podría pensarse que es la razón por la cual muchos de los venezolanos buscan, a través de esas redes de apoyo latinoamericanas, ubicarse cerca a personas que hablen el mismo idioma y que a través de redes de apoyo logren una mejor inserción en ese contexto.

\section{Materiales y métodos}

Esta investigación de corte cualitativo, se ha centrado principalmente en la población ubicada en Florida Central, que más allá del volumen permitiera entender las condiciones de vida de esta población. Si bien esta característica es importante para entender los movimientos migratorios y las realidades de vida de las poblaciones móviles relacionadas con el contexto migratorio, realmente lo que se quiso entender con este trabajo eran las condiciones de salud mental que eran percibidas por las poblaciones que formaron parte de este estudio.

De acuerdo con la Organización Mundial de la Salud (OMS), el estrés está asociado regularmente a la migración, dadas las realidades sociales y culturales de las poblaciones a donde llegan los migrantes en los diferentes destinos; aspectos relacionados como el estatus migratorio, las condiciones de seguridad, la realidad social presente en la migración y la aceptación o rechazo de las poblaciones receptoras pueden generar efectos directos en la salud mental de las poblaciones migrantes (3). 
Por lo anterior, este trabajo buscó principalmente encontrar las implicaciones sociales y culturales de las poblaciones migrantes de venezolanos que se encontraban ubicados en Florida Central, y a partir de allí, establecer las relaciones que la migración en un contexto internacional, lejano al contexto cultural y social latinoamericano, generaba en cierta medida implicaciones en la salud mental y qué tanto esta migración podría o no afectar la salud mental de aquellos que habían buscado en otras latitudes mejores condiciones de vida (migrantes) o proteger su vida (asilados). La mayoría de la población que formó parte de este estudio se encuentra en condición de asilo, por lo cual es importante anotar que este estudio trató también de entender qué tanto estas poblaciones habían recibido asistencia del Estado, en lo que tiene que ver con un tema tan sensible como la salud mental en las poblaciones migrantes que buscan protección del Estado y de aquellos cuya esperanza es mejorar su calidad de vida.

Se seleccionaron personas procedentes de Venezuela que fueran mayores de edad, hombres y mujeres y que quisieran participar de forma libre y voluntaria en el estudio, para lo cual se diseñaron volantes físicos y virtuales explicando el alcance del estudio, y la intencionalidad de conocer las percepciones en torno a la salud mental que tenían las poblaciones migrantes procedentes de Venezuela que estuvieran viviendo en este contexto territorial.

Se aplicaron encuestas, a partir de un cuestionario de 26 preguntas que se dividió en cuatro componentes, a saber: (i) datos generales de la población venezolana, características sociales y demográficas de la población; (ii) datos relacionados con la migración, pretendiendo conocer los motivos de la migración, el momento de la migración y la intensión de quedarse o retornar al lugar de destino; (iii) percepción de sentimientos relacionados con la sociedad receptora, específicamente en cuanto a la acogida, rechazo, discriminación o indiferencia frente a su llegada al entorno receptor; y (iv) aspectos relacionados con sentimientos emocionales, físicos, afectivos y percepciones cognitivas antes, durante o posterior a la migración.

Adicional a lo anterior se profundizó en los datos recolectados por la encuesta, a través de entrevistas grupales (grupos focales) a hombres y mujeres. Se hicieron en total 4 grupos focales, con un total de 41 personas, hombres y mujeres mayores de edad. Estos grupos estuvieron divididos así: 10 personas en Kissimmee (grupo 1), 12 en Orlando (grupo 2), 13 en Orlando (grupo 3) y finalmente 6 personas en Altamont, una subdivisión de Orlando, cerca la ciudad de Apopka (grupo 4). Los participantes que formaron parte de estos grupos viven, tanto cerca de esta zona como de Apopka. Además, se hicieron entrevistas individuales a tres migrantes cuyas historias de vida fueron consideradas importantes para dar aportes a la investigación. Finalmente se realizaron cinco entrevistas a expertos en el tema con el fin de dar a conocer los resultados parciales de esta investigación y comprender las estrategias que en el Estado se habían llevado a cabo. Este último no se incluye en este artículo porque hará parte del informe final que contempla una comparación con el trabajo de campo desarrollado en Cúcuta, con el fin de entender las diferencias entre ambos estudios de caso. 
A todos los participantes el estudio se les solicitó la firma de un consentimiento informado, el cual fue proporcionado por los facilitadores y, una vez, diligenciados, quedaron en custodia del investigador principal. Además, se hicieron grabaciones de audio, las cuales fueron transcritas en Colombia, en las cuales se garantizó el anonimato, para posteriormente ser enviadas a los investigadores del estudio que se encontraban en Estados Unidos (Universidad Central de la Florida). De esta manera, la información de identificación estaba protegida de las leyes de los Estados Unidos, en donde en algún momento estos datos podrían ser requeridos y divulgados por la oficina de Homeland Security. Una vez que se compartieron las transcripciones anonimizadas, fueron cargadas en el programa de análisis cualitativos Dedoose. Después se realizó la codificación con los asistentes de investigación vinculados al proyecto, a través de la convocatoria de Colciencias, mediante el Contrato de financiamiento RC 829 de 2018, firmado entre la Pontificia Universidad Javeriana y Colciencias. Además, se contó con un asistente de investigación proporcionado por la Universidad Central de la Florida, quien domina el español y entendía la metodología; este trabajo fue supervisado por tres de los investigadores que forman parte de este proyecto. Luego, el equipo de investigación generó un libro de códigos y se revisaron todas las transcripciones para verificar su coherencia con estos códigos, de donde surgieron datos relevantes, que son los resultados que se presentan en este artículo.

\section{Resultados}

A continuación, se presentarán los resultados del análisis realizado en los datos recolectados en el trabajo de campo en Florida Central.

\section{Características socioeconómicas de los participantes en el estudio}

Al analizar los datos correspondientes a la conformación de la población correspondiente a sexo, la mayoría de la población encuestada y entrevistada fueron mujeres $(67,5 \%)$ y el grupo minoritario fueron hombres (33,5\%). El promedio de edad entre ambos fue de 45 años, siendo un promedio superior al de la población de venezolanos que han llegado a Colombia, que están entre los 20 y 39 años de edad.

Con respecto al estado civil la mayoría son casados (58\%) que migraron a Estados Unidos como parte de un proyecto familiar, con sus hijos y pareja. Mientras los solteros representan un 16\% y los divorciados, un $13 \%$.

Una característica importante de esta población está relacionada con el nivel educativo, a diferencia de la migración de venezolanos ubicados en el contexto colombiano que, de acuerdo con Migración Colombia, tienen niveles educativos medios y bajos (bachillerato y profesional) (18). En el presente caso de estudio la mayoría son profesionales, y algunos presentan grados de maestría y doctorado ( $20 \%$ y $5 \%$ respectivamente). Lo más importante de este dato es que a pesar de contar con niveles altos de educación, la mayoría de la población no encontró una inserción laboral que correspondiera con su preparación y experiencia profesional, lo que afectó sustancialmente su calidad de vida. 
Otro aspecto importante es el relacionado con el conocimiento del idioma inglés, siendo éste un aspecto importante para encontrar mejores oportunidades laborales en un contexto como Estados Unidos en general y particularmente en Florida. A pesar de ser considerado un Estado hispano hablante, mucha de la oferta laboral exige un nivel alto de inglés. Al indagar este aspecto el $31 \%$ de la población del estudio señaló tener conocimiento del idioma inglés, el resto hablaban otros idiomas como Italiano, Portugués o Alemán, por su estrecha relación con las colonias de europeos que se establecieron en Venezuela, pero que este aspecto generaba una importante al mejoramiento de su calidad de vida, objetivo principal de esta migración.

Precisamente al analizar el poder socioeconómico de esta población, la mayoría de la población reportó haber tenido un alto poder adquisitivo en Venezuela, sin embargo, debido a la devaluación de la moneda y que actualmente hoy en día corresponde a 11.268 bolívares por dólar (26), percibieron un descenso importante en su poder adquisitivo convirtiéndose en el principal motivo por el cual decidieron salir de ese país y buscar mejores condiciones de vida en Florida, Estados Unidos.

Es importante señalar que este descenso socioeconómico se mantuvo con la migración y es posible anotar que debido a la migración hubo una movilidad social descendente ${ }^{1}$ de la mayoría de la población, unido a la pérdida de propiedades en Venezuela, la informalidad en el empleo encontrado en Florida Central y el desconocimiento de sus títulos profesionales. Todo ello generó una pérdida importante de la calidad de vida de esta población, por lo cual se podría señalar que, para este caso - hasta el momento del trabajo de campo de este estudio- estas personas no habían encontrado una mejor calidad de vida en la migración, que adicionalmente se vio afectado por el régimen presente en su país de origen, por lo cual es posible afirmar que, quedándose en Venezuela, probablemente las condiciones no hubieran sido mejores. De acuerdo con la Agencia EFE (26) hoy en día en Venezuela el salario mínimo es 40 mil bolívares, y que al menos 3 millones de trabajadores perciben este ingreso mensual correspondiente a 3,55 dólares, al cual se suma un bono alimenticio que tiene un valor de 25 mil bolívares, lo que representa unos 2,21 dólares.

Al indagar un poco más acerca de los motivos de salida de esta población, de su país de origen (era una pregunta con múltiple respuesta), la mayoría señaló que los factores políticos y sociales fueron el principal motivo por el cual decidieron marcharse del país (85\%), seguido de los factores económicos del propio país y la búsqueda de mejores condiciones oportunidades laborales ( $72 \%$ y $65 \%$ respectivamente). Finalmente, para muchos de los migrantes venezolanos que formaron parte de este estudio, los motivos familiares fue el principal factor por el cual decidieron salir del país (56\%).

Al profundizar en los motivos familiares, se entienden estos como la esperanza de mejorar las condiciones de vida de sus hijos, el poder enviar dinero a familiares, la necesidad de dar aportes a otros familiares (a través de alimentos y ropa). Es importante resaltar que, para muchos de 
los venezolanos, los hijos fueron el motor fundamental de la migración, como lo demuestran los testimonios recogidos en este trabajo, de acuerdo con palabras textuales de algunos de los testimonios:

Mis hijos merecen vivir bien, no tienen la culpa de lo que ha pasado y yo no puedo condenarles a vivir peor, a vivir en un país que no tiene futuro. (Mujer migrante grupo focal realizado en Orlando, 1 de abril de 2019).

Mi hija siempre me dijo, mami yo no quiero seguir acá, mami por favor vámonos de acá. Mi familia es importante y yo no quería salir porque mis papás estaban allí en Barquisimeto, pero debo pensar en mi hija. Mi hija se volvió el motivo de salida de un país que se está resquebrajando que ya no ofrece nada. (Mujer, grupo focal realizado en Kissimmee, 25 de marzo de 2019).

\section{Datos de la salud mental de las poblaciones venezolanas en Central Florida}

Los siguientes datos corresponden principalmente a la percepción de las poblaciones, específicamente con la salud mental y cómo se ha enfrentado a diversas situaciones tales como la soledad, el rechazo o el abandono, aspectos que afectan sin duda la salud mental de las poblaciones en general y de las migrantes en particular.

Al preguntar sobre el estado de cansancio emocional relacionado por el hecho de haber salido de Venezuela, el cual para este trabajo es entendido como el estrés generado por la migración, 4 de cada 5 venezolanos participantes del estudio manifestaron haber sentido estrés. La mayoría de ellos indicaron que el hecho de migrar en las condiciones de incertidumbre y las características forzadas por las cuales tuvieron que salir de su país, les generó estrés y en cierta medida angustia, inclusive por el hecho de enfrentarse a pasos migratorios que no les daban la garantía de la admisión, como en el caso de la migración a Estados Unidos.

La tristeza, la ira y la frustración también estuvieron presentes en el proceso migratorio: 3 de cada 5 participantes, manifestaron haber presentado estas sensaciones. La tristeza estuvo muy relacionada con la desesperanza al dejar un país en condiciones económicas y sociales tan complejas como se encontraba Venezuela a la hora de su partida; la ira se relacionó principalmente con la frustración, relacionada con un sentimiento de impotencia frente a lo ocurrido en su propio país. Todo lo anterior generó sentimientos de vulnerabilidad, tal y como lo manifiesta el siguiente testimonio:

Es ese sentimiento de vulnerabilidad, porque simplemente no estás en control en lo absoluto de nada de lo que está sucediendo, y nosotros no estamos acostumbrados, en Venezuela tenemos familia, tenemos el doctor de toda la vida, el abogado de toda la vida y llegas a un país en donde te sientes sumamente solo y eso pues, produce de todo... Yo viví mi crisis también, eso fue terrible, uno no se imagina lo que es esto (Mujer de 50 años. Grupo Focal Orlando, 1 de abril de 2019).

La soledad fue otro sentimiento que estuvo presente en esta población de venezolanos, pues sintieron que en muchos momentos la soledad está presente, el abandono y esto relacionado con la impotencia de sentirse en un lugar extraño sin el apoyo de un familiar, un amigo o 
alguien (compatriota) que haga parte de ese proceso y con quien se puedan acompañar. En cifras, 4 de cada 5 venezolanos participantes del estudio manifestaron tener sentimientos de soledad durante su estancia en Florida Central. La mayoría de los participantes señalaron que, en muchos momentos, adicional al sentimiento de soledad, también sintieron abandono y rechazo por parte de latinoamericanos que viven en Florida Central. Coincidieron en señalar que las redes establecidas por otros migrantes generaron condiciones de rechazo en contra de ellos en este nuevo contexto migratorio, y que fueron principalmente los compatriotas quienes les brindaron ayuda en este momento en el cual más necesitaron ayuda.

Con respecto a esto último, es importante señalar que 4 de cada 5 participantes del estudio manifestaron haber tenido apoyo de un compatriota en el proceso migratorio. Esto demuestra que los venezolanos han fomentado el sentimiento de solidaridad, y en cierta medida la resiliencia necesaria para superar esta condición a la que se han visto enfrentados. Además, el reconocer lo que les ha sucedido a otros fortalece el sentimiento de resiliencia:

Definitivamente eso, personas con las que tú puedas compartir lo que estás viviendo, saber lo que a otras personas les ha sucedido, cómo las otras personas lo han superado... Yo considero que así se formará un grupo de apoyo en la comunidad para la salud mental. (Mujer migrante venezolana de 35 años. Grupo focal Kissimmee, 25 de marzo de 2019).

Finalmente, con relación los sentimientos y las implicaciones que tiene en su salud mental las condiciones que, actualmente viviendo, la figura 2 muestra de manera resumida las relaciones entre las realidades vividas hoy día por la población migrante venezolana en Florida Central, y las implicaciones que esto ha tenido en su salud mental. 


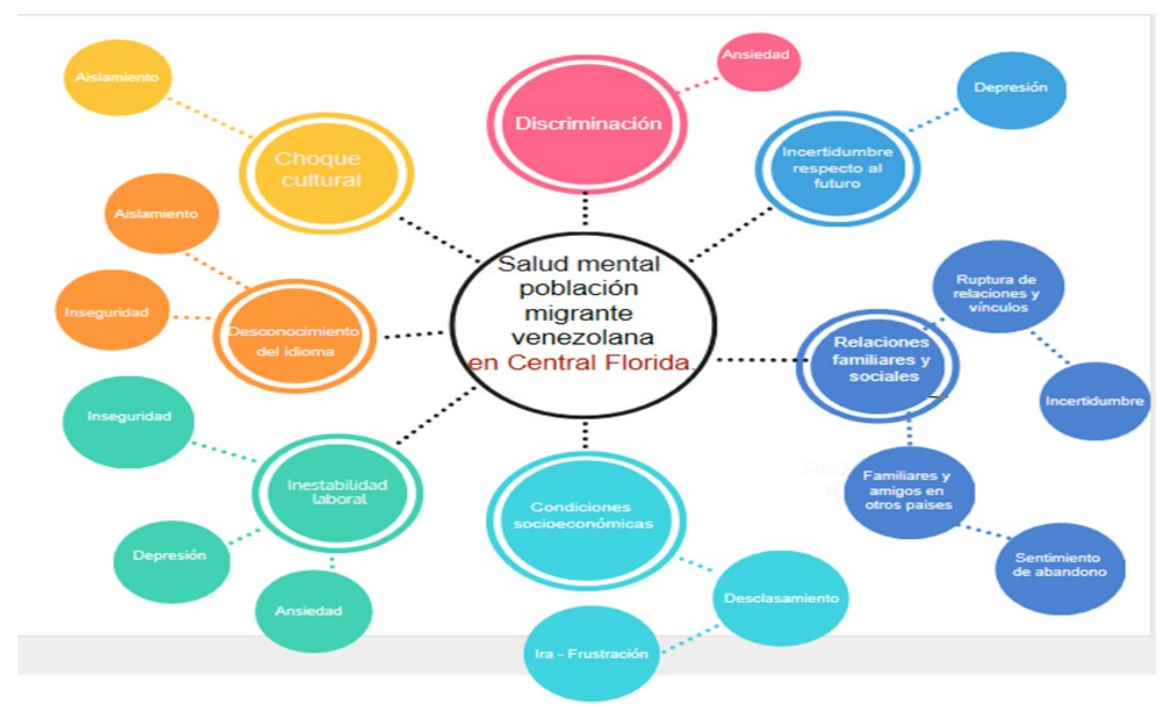

Figura 2 Relaciones entre las realidades vividas por los migrantes venezolanos y las afectaciones percibidas en su salud mental

Fuente: elaboración propia con base en los resultados de los grupos focales a partir de la codificación y análisis cualitativo realizado en Dedoose.

El anterior gráfico trata de mostrar de manera resumida las afectaciones en la salud mental que está presente en la población que formó parte de estudio. Es importante resaltar que -como se señaló con anterioridad- de acuerdo con la OMS (3), la cultura forma parte importante de la salud mental de las poblaciones migrantes. Aquello considerado como el choque cultural (27), los choques culturales están presentes y que para este caso ese choque) ha generado sensaciones de aislamiento. Lo anterior probablemente se debe también a la falta de conocimiento del idioma.

La incertidumbre unida a la inestabilidad laboral, les genera depresión además de ansiedad e inseguridad frente a lo que pueda pasar en un futuro. El desclasamiento originado por la propia migración ha generado ira y frustración a la mayoría de ellos. Finalmente, las relaciones familiares se han reconfigurado, como se aprecia en uno de los testimonios:

Para nosotros, comer en la misma cena, el mismo almuerzo y desayunar lo mismo en grupo de amigos, eso ya nos hace sentir familia (Hombre de 45 años grupo focal realizado en Orlando, 1 de abril de 2019).

La propia reconfiguración les da fortaleza para sentir el apoyo de otros en la misma situación, pero el sentimiento de soledad se relaciona con los dejados atrás, con no poder ofrecer apoyo a los que quedaron atrás, y las reconfiguraciones y desconfiguraciones familiares están presentes. Muchos mantienen contacto transnacional a través de las denominadas redes sociales, el mantener contacto con sus familiares es lo que refuerza el sentimiento de apoyo y, en cierta medida, ayuda a la resiliencia y una mejor condición de salud mental de quienes migraron. Mantener el contacto con los familiares estén donde estén es un gran aporte a la mejora de su salud mental. Sin embargo, lo que pasa con la familia dejada atrás y la incertidumbre que 
ello genera, refuerza el sentimiento de abandono que ellos mismos sienten en el contexto de recepción.

\section{Discusión y conclusiones}

Este trabajo cualitativo muestra las realidades presentes en una población migrante que como la venezolana ha tenido que dejar su territorio y sus condiciones de calidad de vida, en especial por la inestabilidad política y económica. Al no ser una población tradicionalmente migrante, las redes de apoyo parecen no estar presentes, y si bien los apoyos internacionales se los dan los mismos compatriotas, los otros latinoamericanos no se han mostrado solidarios, sino más bien han reforzado el sentimiento de abandono que en muchos casos han generado rechazo en contra de esta población.

Los hijos constituyen el principal factor manifestado por la mayoría de ellos para salir del país. Pero los familiares dejados en Venezuela han marcado la sensación de frustración que les afecta su salud mental, lo que coincide con lo planteado por Ibáñez et al. (28), quienes señalan que la separación de la familia se constituye en una fuente poderosa de estrés aculturativo.

En el presente estudio se hallaron, al igual que en estudios precedentes $(9,13,14,16)$, la existencia de características del entorno del país de acogida que son identificadas por los migrantes venezolanos como condiciones que ellos asocian a algunos síntomas y problemas de salud mental, entre las que se encuentran las diferencias del idioma, la percepción de discriminación e inestabilidad laboral y el deterioro de sus condiciones económicas.

La falta del conocimiento del idioma, unido al descenso de su clase social, ha generado en la migración peores condiciones de vida para muchos de ellos, como han señalado otros estudios $(5,6,29)$. Sin embargo, esa ha sido la mejor opción que han encontrado, frente a la posibilidad de quedarse en Venezuela.

De otro lado, a diferencia de lo que señalan Jurado et al. (10), los hallazgos de este estudio ponen de manifiesto que el mayor nivel educativo no se constituye en un factor protector para la salud mental; en tanto que, para el caso de los migrantes venezolanos en Florida Central, esta característica personal, aunada a las limitaciones de vinculación en el mercado laboral estadounidense en cargos cualificados, puede estar asociada a un descenso en la escala socioeconómica que genera sentimientos de ira y frustración.

La precepción y manifestación de temor, ansiedad, depresión, ira, frustración, aislamiento, inseguridad e incertidumbre que expresaron los participantes en este estudio, pone de manifiesto que ellos identifican y verbalizan algunas manifestaciones emocionales de problemas de estrés aculturativo y problemas de salud mental relacionados con su proceso migratorio; lo cual coincide con lo reportado por Temores-Alcántara et al. (13). 
El estrés, la soledad, la ansiedad y la frustración han estado presentes en el proceso migratorio. El apoyo de los compatriotas ha generado mejores condiciones de vida, y la familia que han ganado en el destino ha sido un gran apoyo para muchos de ellos; lo que coincide con lo reportado en otras investigaciones $(28,29)$.

Algunos estudios realizados internacionalmente muestran que las tasas de incidencia de enfermedades mentales graves y los episodios de psicosis son más altas entre los inmigrantes recientes. Y se ha demostrado (29) que quienes logran adaptarse a nuevos contextos presentan mayores posibilidades de resiliencia, y de sentimientos de esperanza y optimismo.

A pesar de los avances y la evidencia a nivel internacional sobre la importancia de la inversión en salud mental considerada como gasto per cápita de apoyo a la salud mental en toda América, los recursos destinados a este propósito son insuficientes (30). A nivel global, los objetivos de desarrollo sostenible de las Naciones Unidas incluyen un objetivo explícito el cual es "asegurar la salud y promover el bienestar de todos en todas las edades (traducido del inglés)" (2), además de las más de 370 resoluciones sobre salud mental que han sido adoptadas por la ONU (2). Estas acciones apoyan la naturaleza crítica de la atención en salud mental y la relación entre la salud mental y los indicadores clave del bienestar nacional, como la sostenibilidad, la igualdad, la inclusión y la resiliencia, así como la seguridad (2). A pesar de ese énfasis, existen vacíos en la puesta en práctica de la prestación de atención en salud mental, y los datos muestran que las poblaciones son cada vez más vulnerables a factores estresantes y a factores de riesgo, siendo quizá donde se presenta mayor carencia en la prestación de servicios en salud mental.

En lo que tiene que ver con el recurso humano en salud mental, los datos muestran que, en los Estados Unidos, el número medio de profesionales de salud mental en 2017 era 235 por 100.000, en comparación con 9 por 100.000 en Sudamérica (30). Ambos insuficientes para atender a la población. En lo referente al acceso tanto para servicios de salud básica como para salud mental es limitado, y esta limitación es mayor en aquellos que no tienen seguros de salud.

Históricamente, los inmigrantes que llegaban a Estados Unidos eran elegibles para acceder a opciones de cobertura pública y privada en salud, pero los recientes cambios de política han afectado el panorama (31). Han aumentado las tasas de inmigrantes no asegurados en el país, ya que el temor con respecto a las implicaciones legales para el estatus migratorio ha llevado a las personas a evitar el uso de cualquier opción pública, tales como los servicios de Medicaid, para los que podrían ser elegibles, y que, además, les podrían proporcionar prevención y atención en situaciones de salud mental agudas. De acuerdo con Parmet (31):

la pobreza desproporcionada, el no pertenecer a la población blanca y no hablar inglés, son aspectos que están mucho más presentes en las poblaciones vulnerables en Estados Unidos. Consecuentemente, estas poblaciones son quienes perciben las ineficiencias y los mayores vacíos en el sistema de salud en Estados Unidos (31, blog). 
En los últimos años, las acciones de política han restringido la capacidad de comprar seguros de salud, incluso en los mercados privados, para aquellos que están en el país sin documentación, así como para aquellos que están cubiertos por la Acción Diferida para los llegados en la infancia o DACA por su sigla en inglés (Deferred Action for Childhood Arrivals).

Aunque los centros de salud frecuentemente atienden a estas poblaciones, los fondos federales siguen en riesgo, dejando amplias brechas en la disponibilidad de servicios (31). A pesar de estas condiciones, se presentan vacíos en la capacidad de acceso a los servicios, la necesidad sigue estando presente, y el riesgo para la salud pública continúa siendo un problema que requiere atención. El consenso mundial sobre las necesidades de atención de la salud mental es un llamado a la acción para abordar estas barreras estructurales y sistémicas a los servicios, tal y como quedó consignado en el plan para la atención en salud mental para las poblaciones 2015-2020 de la OMS (32).

Este trabajo demuestra que es necesario indagar mucho más en la relación de migración y salud mental, y comparar los resultados de este estudio con otros casos de poblaciones que tradicionalmente han sido migrantes, con el fin de encontrar diferencias y similitudes para fortalecer las mejores condiciones de salud mental que den aportes a programas que atiendan realidades diferenciales, reconociendo particularidades nacionales, pues no por ser considerados como hispanos es posible atender a todos de la misma manera; clamor de la población que formó parte de este estudio.

\section{Referencias}

1. Programa de las Naciones Unidas para el Desarrollo (PNUN). Objetivos de desarrollo sostenible. New York: PNUD; 2010. https://www.undp.org/content/undp/es/home/sustainable-development-goals.ht $\mathrm{ml}$

2. Izutsu T, Tsutsumi A, Minas H, Thornicroft G, Patel V, Ito A. Mental health and wellbeing in the Sustainable Development Goals. Lancet Psychiat. 2015;2(12): 1052-1054. https://doi.org/10.1016/S 2215-0366(15)00457-5

3. Organización Mundial de la Salud. Promoción de la salud de los migrantes. Informe de la Secretaría. OMS. 12 de diciembre de 2016. EB140/24. http://apps.who.int/gb/ebwha/pdf_files/EB140/B140_2 4-sp.pdf

4. Alegría M, Álvarez K, DiMarzio K. Immigration and mental health. Curr Epidemiol Rep. 2017;4(2): 145-155. https://doi.org/10.1007/s40471-017-0111-2

5. Virupaksha HG, Kumar A, Nirmala BP. Migration and mental health: An interface. J Nat Sci Biol Med. 2014;5(2): 233-239. https://doi.org/10.4103/0976-9668.136141 
Migración de venezolanos a Florida Central, Estados Unidos. Aspectos relacionados con la percepción de condiciones y necesidades de salud mental en 2019

6. Li M. Pre-migration trauma and post-migration stressors for Asian and Latino American immigrants: Transnational stress proliferation. Social Indicators Research. 2016; 129(1): 47-59. https://doi.org/1 $0.1007 / \mathrm{s} 11205-015-1090-7$

7. Pumariega AJ, Rothe E, Pumariega JB. Mental health of immigrants and refugees. Community Ment Health J. 2005;41(5): 581-597. https://doi.org/10.1007/s10597-005-6363-1

8. Virupaksha HG, Ashok K, Bergai PN. Migration and mental health: An interface. J Nat Sci Biol Med. 2014;5(2): 233. https://doi.org/10.4103/0976-9668.136141

9. Bojorquez, I. Salud mental y migración internacional [editorial]. Rev. Univ. Ind. Sant. Salud. 2015;47(2): 111-113. http://www.scielo.org.co/pdf/suis/v47n2/v47n2a01.pdf

10. Fernández AC, Mandujano JL, Guzmán EF. Salud y enfermedad en los migrantes internacionales México-Estados Unidos. Ra Ximhai. 2014;10(1): 291-306. http://www.redalyc.org/articulo.oa?id=4 6129579013

11. Salaberria Irízar K, Haro S, del Valle A. Estrés migratorio y salud mental. Behav Psychol. 2017;25(2): 419-432. https://pesquisa.bvsalud.org/portal/resource/pt/ibc-167109

12. Collazos F, Ghali K, Ramos M, Qureshi A. Salud mental de la población inmigrante en España. Rev Esp Salud Pública. 2014;88(6): 755-761. http://dx.doi.org/10.4321/S1135-57272014000600008

13. Jurado D, Alarcón RD, Martínez-Ortega JM, Mendieta-Marichal Y, Gutiérrez-Rojas L, Gurpegui, M. Factores asociados a malestar psicológico o trastornos mentales comunes en poblaciones migrantes a lo largo del mundo. Revista de Psiquiatr Salud Ment. 2017;10(1): 45-58. http://dx.doi.org/10.101 6/j.rpsm.2016.04.004

14. Urzúa MA, Boudon Torrealba S, Caqueo-Urízar A. Salud mental y estrategias de aculturación en inmigrantes colombianos y peruanos en el norte de Chile. Acta Colombiana de Psicología. 2017;20(1): 70-79. https://doi.org/10.14718/ACP.2017.20.1.5

15. Cabieses B, Gálvez P, Ajraz N. Migración internacional y salud: el aporte de las teorías sociales migratorias a las decisiones en salud pública. Rev Peru Med Exp Salud Publica. 2018;35(2): 285-291. https://doi.org/10.17843/rpmesp.2018.352.3102

16. Temores-Alcántara G, Infante C, Caballero M, Flores-Palacios F, Santillanes-Allande, N. Salud mental de migrantes centroamericanos indocumentados en tránsito por la frontera sur de México. Salud Publica Mex. 2015;57(3): 227-233. http://dx.doi.org/10.21149/spm.v57i3.7561

17. Garrido HM. Las 5 causas y 10 efectos de la crisis del petróleo: ¿quién gana, quién pierde y cómo nos afecta? 20 Minutos [Internet]. Martes 26 de enero de 2016. https://www.20minutos.es/noticia/26531 91/0/causas-efectos/crisis-petroleo-barato/perjudicados-beneficiados/

18. Migración Colombia. Radiografía de venezolanos en Colombia. [Internet]. 2019. Disponible en: http:/ /www.migracioncolombia.gov.co/venezuela/radiografia_web.pdf

19. Caicedo Cardona, JM. Colombia y Venezuela. Más que 2200 kilómetros de frontera. 12 de abril de 2019. https://www.comminit.com/la/content/colombia-venezuela-m\%C3\%A1s-que-2200-kil\%C3\% B3metros-de-frontera

20. Consejo Nacional de Política Económica y Social de Colombia (CONPES). CONPES 3950: Estrategia para la atención de la migración desde Venezuela, 23 de noviembre de 2018. Bogotá: 
DNP. https://www.dnp.gov.co/Paginas/CONPES-definio-la-Estrategia-para-la-Atencion-de-la-Migr acion-desde-Venezuela.aspx

21. Mossaad, N. Annual Flow Report. Refugees and Asylees: 2018. U.S. Homeland Security Homeland Security. Marzo de 2019. https://www.dhs.gov/sites/default/files/images/OIS/2018/refugees_asylee s_2018.pdf

22. Mossaad, N. Annual Flow Report Homeland Security Homeland Security. Marzo de 2019. https://ww w.dhs.gov/sites/default/files/publications/Refugees_Asylees_2017.pdf

23. Migración Colombia. Infografía PEP 1er semestre 2018. https://www.migracioncolombia.gov.co/infog rafias/230-categorias-migratorias/infografia-pep-primer-semestre

24. O'Toole M. Venezuela, now a top source of U.S. asylum claims, poses a challenge for Trump. Los Ángeles Times, 5 de junio de 2019. https://www.latimes.com/politics/la-na-pol-trump-venezuela-as ylum-immigration-20190605-story.html

25. U.S. Immigration and customs enforcement. ICE Enforcement and removal operations Report. Fiscal Year 2018. https://www.ice.gov/doclib/about/offices/ero/pdf/eroFY2018Report.pdf

26. Agencia EFE. La insoportable devaluación del bolívar. Portafolio. 1 de agosto de 2019. https://www.p ortafolio.co/internacional/sigue-la-devaluacion-del-bolivar-532156

27. Bulcourf P. Samuel P. Huntington. El choque de civilizaciones y la reconfiguración del orden mundial. Barcelona: Paidós; 1997.

28. Ibáñez GE, Dillon F, Sánchez M, De La Rosa M, Tan L, Villar ME. Changes in family cohesion and acculturative stress among recent Latino immigrants. J Ethn Cult Divers Soc. Work. 2015;24(3): 219-234. https://doi.org/10.1080/15313204.2014.991979

29. Kirmayer LJ, Narasiah L, Munoz M, Rashid M, Ryder A G, Guzder J, et al. Common mental health problems in immigrants and refugees: General approach in primary care. CMAJ. 2011;183(12): 959-967. https://doi.org/10.1503/cmaj.090292

30. Pan American Health Organization (PAHO). Atlas of Mental Health of the Americas 2017. Washington DC: PAHO; 2018. http://iris.paho.org/xmlui/handle/123456789/49664

31. Parmet W. Immigration and health care under the Trump administration. Health Affairs Blog. 18 de enero de 2018. https://www.healthaffairs.org/do/10.1377/hblog20180105.259433/full/

32. Organización Panamericana de la Salud (OPS) y Organización Mundial de la Salud. Plan de Acción sobre Salud Mental 2015-2020. 3 de octubre del 2014. http://www.paho.org/hq/index.php?option=com_content\&view=article\&id=11337\%3Aplanof-action-on-mental-health-2015-2020\&catid=8190\%3Ageneral\&Itemid=41600\&lang=es

Notas

Artículo de investigación.

Se refiere a la forma de movilidad social que consiste en que una persona pase de una clase social o un estatus económico alto a otro inferior. La movilidad social descendente provoca una pérdida de estatus social. 